\title{
Time-dependent energetic proton acceleration and scaling laws in ultra-intense laser pulses interactions with thin foils
}

\author{
Yongsheng Huang, ${ }^{1,2, *}$ Yuanjie Bi, ${ }^{1,2}$ Yijin Shi, ${ }^{1}$ Naiyan Wang, ${ }^{1}$ Xiuzhang Tang, ${ }^{1}$ and Zhe $\mathrm{Gao}^{2}$ \\ ${ }^{1}$ China Institute of Atomic Energy, Beijing 102413, China. \\ ${ }^{2}$ Department of Engineering Physics, Tsinghua University, Beijing 100084, China.
}

(Dated: November 5, 2018)

\begin{abstract}
A two-phase model, where the plasma expansion is an isothermal one when laser irradiates and a following adiabatic one after laser ends, has been proposed to predict the maximum energy of the proton beams induced in the ultra-intense laser-foil interactions. The hot-electron recirculation in the ultra-intense laser-solid interactions has been accounted in and described by the time-dependent hot-electron density continuously in this model. The dilution effect of electron density as electrons recirculate and spread laterally has been considered. With our model, the scaling laws of maximum ion energy have been achieved and the dependence of the scaling coefficients on laser intensity, pulse duration and target thickness have been obtained. Some interesting results have been predicted: the adiabatic expansion is an important process of the ion acceleration and cannot be neglected; the whole acceleration time is about $10-20$ times of laser pulse duration; the larger the laser intensity, the more sensitive the maximum ion energy to the change of focus radius, and so on.

PACS numbers: 52.38.Kd,41.75.Jv,52.40.Kh,52.65.-y
\end{abstract}

*Electronic address: hyc05@mails.tsinghua.edu.cn 


\section{INTRODUCTION}

Proton acceleration mechanisms in ultra-intense laser pulses interaction with thin solid targets attract more and more interest nowadays [1, 2, 3]. Various models [2, 4, 5, 6] have been presented to estimate the maximum energy of proton beams. However, the models given by Wilks et al. , Kaluza et al., Schreiber et al. and Fuchs et al. are all based on isothermal expansions of quasineutral plasmas [7]. Robson et al. presented a two-phase temperature-varying model, where the hot-electron temperature first increases linearly on the pulse duration timescale and then decreases adiabatically with time . However, in the pulse duration, does the hot-electron temperature rise up linearly? That is still difficult to be validated. Generally ones assume that, when an ultraintense laser pulse interacts with a solid target, the laser-produced fast electrons with a uniform temperature, $k_{B} T_{e}$, determined by the laser ponderomotive potential are instantly created in front of the target and propagate through the target collisionlessly and then form a high energy plasma at the rear of the target. When the laser pulse still exists, the hot-electron temperature, $k_{B} T_{e}=$ $m_{e} c^{2}(\gamma-1)$, is assumed invariant due to a constant energy supply from the laser pulse, where $\gamma=\left(1+I \lambda^{2} / 1.37\right)^{0.5}$ is the relativistic factor, $I$ is the laser intensity in $10^{18} \mathrm{~W} / \mathrm{cm}^{2}, \lambda$ is the laser wave length in $\mu \mathrm{m}, m_{e}$ is the electron mass and $t_{l}$ is the pulse duration. The plasma expansion is an isothermal expansion. Therefore, a two-phase model different from Robson et al. is proposed in this article, where the plasma expansion is isothermal in the laser pulse duration and then the hot-electron temperature decreases as $\left(t / t_{l}\right)^{-(1+1 / \gamma)}[8]$.

The electron density distribution satisfies Boltzmann relationship: $n_{e}=n_{e 0} \exp \left(e \phi / k_{B} T_{e}\right)$ and $n_{e 0}$ stays a constant and time-independent in the previous models [2, 4, 5, 7] without hot-electron recirculation, where $e$ is the elementary charge and $\phi$ is the electric potential. Therefore, with a little adjustment of some parameters: the acceleration time [4, 5] , the opening angle of electrons [2] and electron density, $n_{e 0}$ [2, 4, 5], Mora's result can be used to estimate the maximum energy of proton beams for thick targets, where the influence of hot-electron recirculation on the ion acceleration can be ignored. Although Robson et al. have presented a two-phase model which is consistent with experiments, the hot-electron recirculation is still ignored. However, Mackinnon et al. [9] observed enhancement of proton acceleration by hot-electron recirculation in thin foils whose thickness is less than a critical value. In addition, Sentoku et al. [10] predicted an equation to conclude the influence of electron recirculation and proved the hot-electron recirculation cannot be ignored in the laser-foil interactions, although they didn't propose a clear description of electron 
recirculation and their physical picture is too simple and not clear. The assumption: the maximum hot-electron density for a thin foil is a constant and $\mathrm{N}$ times of the value for a critical target thickness in Sentoku et al.'s model, is rough and unreasonable. Because there are n times of the electron recirculation they happen one after the other and the electron density can't jump to $n$ times of the initial density. After that, Huang et al.[11] presented a step model to describe the influence of the hot-electron recirculation on the laser-ion acceleration. In the step model, the hot-electron density rises step by step with isothermal plasma expansions. In fact, the electron density should rise continuously and then decrease to zero as the time tends to infinite. Therefore, the time-dependent hot-electron density and the electric field are necessary for the description of the hot-electron recirculation and the whole process of the ion acceleration. The dilution effect of the electron density as the electrons circulate and spread laterally should be considered but not accounted in the previous models $[10,11]$.

In Sec. II, a new two-phase model which contains three-dimensional effect (the thickness effect, the angular effect, which are discussed in detail by Huang et al. [11], and the dilution effect as the electrons circulate and spread laterally) and the hot-electron recirculation is proposed, where the plasma expansion is isothermal in the pulse duration and then adiabatic. The main processes of our model are two: first, combining the Mora's result in ref. [7] and the increase of the electron density in the pulse duration, with the assumption: the hot-electron temperature is a constant, the dependence hot-electron density, the electric field and the ion velocity on the time are obtained; second, with the assumption of an adiabatic expansion, the dependence of the temperature of hot electrons on time as proposed by Mora[8] has been used and then the maximum ion velocity is obtained easily. A most significant progress of our model is that: the time-dependent electric field and hot-electron density can be given easily by solving two nonlinear equations. As a result of the model, the duration of the time-dependent electric field at the ion front is approximately one to two times of the main laser pulse duration which is consistent with the result presented by d'Humieres, Lefebvre, Gremillet, and Malka in [3] using the particle-in-cell (PIC) simulation. The whole acceleration time is about $10-20$ times of the laser pulse duration. And we also proofed that the adiabatic expansion is an important process for the ion acceleration and cannot be neglected. Our model can be used in the same application content as Robson's model: the target normal sheath acceleration of ions, however, from the above discussions, it is more reasonable and easily to use than their's.

In Sec. III, with a proper laser absorption efficiency for thick targets, our two-phase model has 
been compared with experiments and they are consistent as shown in Table The laser absorption stays constant with the target thickness for thick target. The laser absorption efficiency for the target of arbitrary thickness has been calculated by particle-in-cell (PIC) simulations [3], although there is no analytic result of that. With the laser absorption efficiency of $40 \%$ for the target of $3 \mu \mathrm{m}$ given by the result of PIC simulations, the comparison between our model and the experimental result is shown in Table I] If the laser absorption is known, for the target of arbitrary thickness, the maximum energy of proton beams and time-dependent electric field and electron density can all be obtained using our model.

In Sec. IV] with our two-phase model, the scaling law of maximum ion energy with respect to laser intensity for a series of constant pulse duration has been given and discussed as shown in Figures 2. The dependence of maximum ion energy on target thickness, focus radius, laser pulse duration have been obtained. With the scaling law, some interesting results have been obtained and discussed in detail. Also in this section, the limits of our model have been discussed.

\section{TIME-DEPENDENT ENERGY PROTON ACCELERATION}

For convenience, the physical parameters: the time, $t$, the ion position, $l$, the ion velocity, $v$, the electron field, $E$, the hot-electron density, $n$, and the light speed, $c$, are normalized as Equation (1)

in [11]. Then the normalized parameters are : $\tau, \hat{l}, u, \hat{E}, \hat{n}, \hat{c}$ as shown by Equation (1) in [11].

When an ultra-intense laser pulse interacts with a solid target, the laser-produced fast electrons with a uniform temperature, $k_{B} T_{e}$, determined by the laser ponderomotive potential are instantly created in front of the target and propagate through the target and then form a high energy plasma at the rear of the target. Here, it is assumed that the hot electron transport is collisionless, which is true for high energetic electrons, thin foils or the atomic number of the materials of the target is low. Hot electrons at the rear of the target can be considered to be reflected by sheath field at the ion front [7, 12] and come back to the front of the target, because the field there is strongest. Once hot electrons are created, they will bounce between the ion front before the target and the ion front at the rear side. Since we consider the electron motion is collisionless, the bounce of hot electrons will last in the whole time of the plasma expansion. When the hot electrons propagate through the target, the electron beam can be assumed to be in equilibrium. 


\section{A. Isothermal Expansion}

The hot-electron speed used is the light speed $c$. Here the choice of $t=0$ is the same as that in the step model given by Huang and co-workers in [11]. For simplicity in the $-L / c \leq t \leq t_{l}-L / c$, where $L$ is the target thickness, the laser intensity is assumed to be a constant, therefore, the hotelectron temperature, $k_{B} T_{e}=m_{e} c^{2}(\gamma-1)$, is invariant. The plasma expansion is an isothermal expansion.

The fast-electron density is a function of the parameters: the acceleration time, $\tau$, the target thickness, $L$, laser intensity, $I$, laser focus radius, $r_{L}$, the laser absorption efficiency, $\eta$, the incidence angle of the laser pulse, $\theta_{i n}$, the half-opening angle of fast electrons, $\theta_{e}$. The time-dependent electron density is assumed:

$$
\begin{gathered}
n_{e}\left(\tau, L, I, r_{L}, \eta, \theta_{i n}, \theta_{e}\right)=N(\tau, L) n_{e 0}\left(L, I, r_{L}, \eta, \theta_{i n}, \theta_{e}\right), \\
N(\tau, L)=1, \tau=\tau_{1}=\tau_{L},
\end{gathered}
$$

where $\tau_{1}$ is the time when the zeroth hot-electron recirculation ends and hot electrons go forth to reach the rear of the target the second time, $\tau_{L}=2 \hat{L} / c / \sqrt{2 e}$, here $e$ denotes the numerical constant $2.71828 \ldots . . n_{e 0}\left(L, I, r_{L}, \eta, \theta_{i n}, \theta_{e}\right)$ is the hot-electron density when hot electrons return back from the ion front before the target and go forth to reach the rear of the target the second time and $N(\tau, L)$ describes the increase of the maximum electron density due to electron recirculation and the electron generation by the laser-plasma interactions at the front of the target.

Using Eq. (2) in [2], since the total number of hot electrons that propagate through the target at $t=t_{L}=2 L / c, N_{e}=\eta(L) E_{l} /\left(k_{B} T_{e}\right)$ for $t_{l} \leq t_{L}$ and $N_{e}=\eta(L) E_{l} t_{L} /\left(k_{B} T_{e} t_{l}\right)$ for $t_{l} \geq t_{L}, n_{e 0}$ in Eq. (1) can be estimated by:

$$
\begin{gathered}
n_{e 0}=\frac{4.077 \eta(L) I_{10^{18} \mathrm{~W} / \mathrm{cm}^{2}}}{(\gamma-1)\left(1+\left(L^{*} / r_{L}\right) \tan \left(\theta_{\mathrm{e}}\right)\right)^{2}}, t_{l} \geq t_{L} \\
n_{e 0}=\frac{4.077 \eta(L) I_{10^{18} \mathrm{~W} / \mathrm{cm}^{2} t_{l}}}{(\gamma-1)\left(1+\left(L^{*} / r_{L}\right) \tan \left(\theta_{\mathrm{e}}\right)\right)^{2} \mathrm{t}_{\mathrm{L}}}, t_{l} \leq t_{L},
\end{gathered}
$$

where $r_{L}$ is the laser pulse focus radius, $L^{*}=L / \cos \left(\theta_{i n}\right)$ is the efficient target thickness, $\theta_{i n}$ is the incidence angle of the laser pulse and $\theta_{e} \approx 17^{\circ}$ is half-opening angle of the superathermal electrons which was measured by Santos et al. [12]. With Eq. (2) and Eq. (3), the three-dimensional effect has been accounted in through the considering of the half-opening angle of electrons, $\theta_{e} \approx 17^{\circ}$. Note that the right side of Eq. (3) has a factor, $t_{l} / t_{L}$, which is not in the right side of Eq. (2). For $t_{l} \leq t_{L}, N_{e}=\eta(L) E_{l} /\left(k_{B} T_{e}\right)$, where $E_{l}$ is the energy of laser pulse. However, for $t_{l} \geq t_{L}$, at $t=t_{L}$, hot electrons are still being generated by the laser-plasma interactions at the front of the 


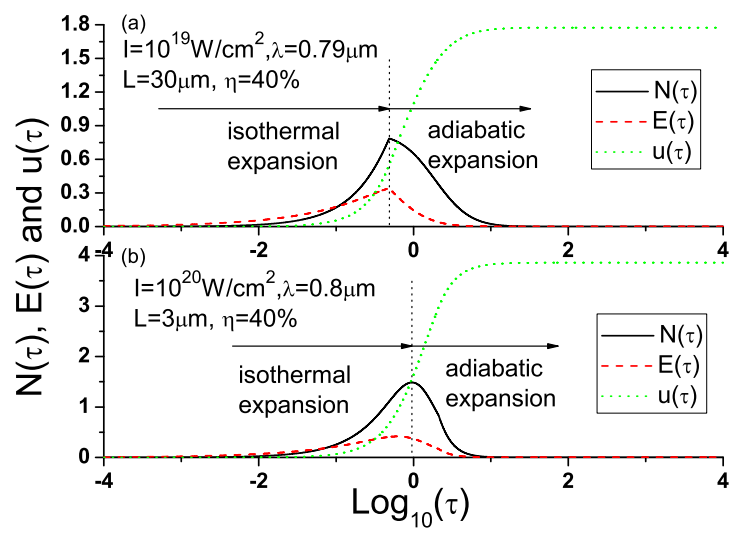

FIG. 1: (Color online) The time-dependent hot-electron density, acceleration field and the speed of ions versus $\tau=\omega_{p i} t / \sqrt{2 e}$ given by the Time-Dependent Target Normal Sheath Acceleration for $\theta_{i n}=30^{\circ}$ (in Fig. 1(a)), $\theta_{\text {in }}=22^{\circ}$ (in Fig. 1(b)), $\theta_{e}=17^{\circ}$ and $\eta=40 \%$. In Figure 1(a), the laser pulse parameters are $I=1.0 \times 10^{19} \mathrm{~W} / \mathrm{cm}^{2}, \lambda=790 \mathrm{~nm}, r_{L}=2.5 \mu \mathrm{m}, L=30 \mu \mathrm{m}$ and $t_{l}=150 \mathrm{fs}$. In Figure $1 \mathrm{~b}$ ), the laser pulse parameters are $I=1.0 \times 10^{20} \mathrm{~W} / \mathrm{cm}^{2}, \lambda=800 \mathrm{~nm}, r_{L}=2.5 \mu \mathrm{m}, L=3 \mu \mathrm{m}$ and $t_{l}=100 \mathrm{fs}$.

target, and the number of hot electrons which propagate through the target is a part of the total number, $N_{e}=\eta(L) E_{l} t_{L} /\left(k_{B} T_{e} t_{l}\right)$. Therefore, Equations (2) and (3) are obtained. When $r_{L} \gg L$ and $\tan \left(\theta_{i n}\right) \ll 1,\left(1+\left(L^{*} / r_{L}\right) \tan \left(\theta_{\text {in }}\right)\right)^{2} \approx 1$, the angular effect can be neglected. Therefore, the influence of $\eta(\hat{L})$ and electron recirculation become dominated for thin targets. For example, for $I=3 \times 10^{20} \mathrm{~W} / \mathrm{cm}^{2}, \lambda=1.053 \mathrm{~nm}$ and $t_{l}=500 \mathrm{fs}$ [13], the temperature of hot electrons is about $5.5 \mathrm{MeV}$. For $\eta(L)=50 \%, r_{L}=5 \mu \mathrm{m}$, with Eq. (3), the electron density is about $4.8 \times 10^{19} \mathrm{~cm}^{-3}$ and it is about the value measured by x-ray in [13]. Since the laser pulse duration is $t_{l}$, with similar discussion in [11], the critical target thickness for the hot-electron recirculation is: $L_{c}=0.5 c t_{l}$.

With reference to the discussion and method given by Huang and co-workers in [11], the relationship between the ion velocity at the ion front and the electron density can be described by Equation (12) in [11]. With that equation, the ion velocity is decided by $N(\tau)$. Therefore, the solution of $N(\tau)$ is a key point. Although it has been given by Huang et al. [11] with a simple model, it is rough for three reasons: (1) it is discrete, however the actual electron density change continuously; (2) the electron density deceases as electrons recirculate and spread laterally and this dilute effect was not counted in the Step Model[11]; (3) the turning point of hot electrons at front of the target is not static but moving with the expansion of the plasma too. In this paper, a more actual and valuable method will be proposed to calculate a continuous solution of $N(\tau)$ as follows. 
Assuming that: the velocity at the ion front before the target is the same as that at the rear of the target and the two turning points for the electron recirculation are the ion front before the target and at the rear respectively, if hot electrons satisfy uniform distribution in the bulk from the ion front before the target to the ion front at the rear, $N(\tau)$ is decided by

$$
N(\tau)=\frac{\int_{-\hat{L} / \hat{c}}^{\tau-\hat{L} / \hat{c}} f d \tau}{\int_{-\hat{L} / \hat{c}}^{\tau_{L}-\hat{L} / \hat{c}} f d \tau} \frac{\hat{L}+2 \hat{l}\left(\tau_{L}\right)}{2 \hat{l}(\tau)+\hat{L}} F_{\theta, 3-D}, \tau \leq \tau_{l},
$$

where $f$ represents the generation rate of hot electrons in the interaction of laser pulses with the plasma at the front of the target, $F_{\theta, 3-D}=\left\{\frac{f_{\theta}\left[\hat{L}+2 \hat{l}\left(\tau_{L}\right)\right]+\hat{r}_{L}}{f_{\theta}[\hat{L}+2 \hat{l}(\tau)]+\hat{r}_{L}}\right\}^{2}$ and $f_{\theta}=\tan (\theta) / \cos \left(\theta_{i n}\right) . f$ depends on the absorption mechanisms of laser pulses and decides the density of hot electrons. The factor, $F_{\theta, 3-D}$, corresponds to the decrease of on-axis density as hot electrons circulate and spread laterally with an given opening angle, $\theta_{e}$. And also it reflects three-dimensional effect on the ion acceleration. The special integrating limits are because there is an interval before the electrons generated by the laser pulse at the front of the target come to the rear. With the assumption: $f=\bar{f}$ for $\tau \in\left[0, \tau_{l}\right]$, Eq. (4) can be simplified to be:

$$
N(\tau)=\frac{\tau}{\tau_{L}} \frac{\hat{L}+2 \hat{l}\left(\tau_{L}\right)}{2 \hat{l}(\tau)+\hat{L}} F_{\theta, 3-D}, \tau \leq \tau_{l},
$$

\section{B. Adiabatic Expansion}

When $t \geq t_{l}$, the laser pulse has gone and the acceleration field at the ion front decreases quickly for two reasons. First, the temperature of hot electrons decreases with time as shown by[8]:

$$
T_{e} \propto\left(\tau / \tau_{l}\right)^{-(1+1 / \gamma)}
$$

For the nonrelativistic case, $\gamma=1$, with Eq. (6), $T_{e} \propto t^{-2}$ which is consistent with all the previous work of adiabatic expansion into a vacuum[14, 15, 16] . For the ultra-relativistic case, $\gamma \longrightarrow+\infty$, with Eq. (6), $T_{e} \propto t^{-1}$ which is the same with Mora's results[8]. After the laser pulse vanishes, the ion front does not stop and the electron bulk still increases. Therefore, the electron density, $N(\tau)$, decreases as given by:

$$
N(\tau)=\frac{\tau_{l}}{\tau_{L}} \frac{\hat{L}+2 \hat{l}\left(\tau_{L}\right)}{2 \hat{l}(\tau)+\hat{L}} F_{\theta, 3-D}, \tau \geq \tau_{l} .
$$

Eq. (5) and Eq. (7) are all nonlinear differential equations and have no analytic solutions. However, the numerical results can be obtained by computer with iterative method. The initial 
$N(\tau)$ is given by the solution of the Eq. (5) and Eq. (7) in which $F_{\theta, 3-D} \equiv 1$. As an example, the solutions of a thin foil and a thick solid target have been given by Figure 1, Figure 1(a) corresponds a thick target of $30 \mu \mathrm{m}$ and the hot electron recirculation can be ignored. Figure 1(b) corresponds a thin foil of $3 \mu \mathrm{m}$ and the maximum value of $N(\tau)$ is about 1.5 , which is lower than that given by Huang and co-workers in [11] and Sentoku et al. in [10] and reflects the three-dimensional effect. From Fig. 1, some interesting results can be obtained:

(1) The whole accelerate time of ions is about $10-20$ times of the laser pulse duration. After that, the separating field is close to zero and the acceleration ends.

(2) The electron density and the electric field reach their maximum value at the time $t=t_{l}$ as expected by our discussion and the gain energy of ions in the process of the isothermal expansion is approximately a quarter of the finally energy. Therefore, the adiabatic expansion is also important for the ion acceleration although the electron density and electric field decrease in this process.

(3) The influence of the hot-electron recirculation on the ion acceleration for thin foils is obvious. The maximum ion energy for thin foils is larger than that for thick targets.

With solutions of $N(\tau)$, the time-dependent electric field and the ion velocity at the ion front can be obtained. Therefore, for the target of arbitrary thickness, the maximum energy of proton beams can be achieved if the laser absorption efficiency is known.

\section{COMPARISON WITH EXPERIMENTS}

Our time-dependent model is compared with experiments, the results are listed in Table \.

For example, for $I=1 \times 10^{20} \mathrm{~W} / \mathrm{cm}^{2}, \lambda=0.8 \mathrm{~nm}$ and $t_{l}=100 \mathrm{fs}$, the critical target thickness is about $15 \mu \mathrm{m}$, according to Machinnon et al. [9] , $E_{\max }(L=30 \mu \mathrm{m})=6.2 \mathrm{MeV}$. With the simulation results (Figure 12 in [3]), the laser absorption stays constant of about $35 \% \rightarrow 50 \%$ with the target thickness for thick target, $L \gtrsim 1 \mu \mathrm{m}$. The laser absorption changes with target thickness, the contrast ratio between the main pulse and prepulse and the prepulse duration [2, 11] for $L \lesssim 1 \mu \mathrm{m}$. For different target thickness, the permeation of laser pulse is different. For different contrast ratio and prepulse duration, the scale length of the preplasma is different, which induces different laser absorption mechanism. Therefore, the laser absorption efficiency, $\eta(L)$, is different and difficult to be assured. Different $\eta(L)$ corresponds to different electron density, $n_{e 0}$. The plasma frequency and acceleration parameters depend on $n_{e 0}$. After all, the changing law of $\eta(L)$ with $L$ for $L \leq 1 \mu \mathrm{m}$ is quite important for the proton acceleration and still a challenge. Without $\eta(L)$, our model can not 
TABLE I: This is a comparison between our two-phase model and some experiments for $\theta_{\text {in }}=30^{\circ}$ in ref. [2], $\theta_{\text {in }}=22^{\circ}$ in ref. [9] and $\theta_{e}=17^{\circ}[12]$.

\begin{tabular}{|c|c|c|c|c|c|c|c|c|}
\hline $\begin{array}{c}I \\
\left(10^{18} \mathrm{~W} / \mathrm{cm}^{2}\right)\end{array}$ & $\begin{array}{c}\lambda \\
(\mu \mathrm{m})\end{array}$ & $\begin{array}{c}t_{l} \\
(\mathrm{fs})\end{array}$ & $\begin{array}{c}r_{L} \\
(\mu \mathrm{m})\end{array}$ & $\begin{array}{c}L \\
(\mu \mathrm{m})\end{array}$ & $\begin{array}{l}\eta(L) \\
(\%)\end{array}$ & $\begin{array}{c}n_{e 0}(L) \\
\left(10^{20} / \mathrm{cm}^{3}\right)\end{array}$ & $\begin{array}{c}E_{\text {max }} \text { from experiments } \\
\text { or PIC }(\mathrm{MeV})\end{array}$ & $\begin{array}{c}E_{\text {max }} \text { from our Model } \\
(\mathrm{MeV})\end{array}$ \\
\hline 10 & 0.79 & 150 & 2.5 & 30 & 40 & 0.33 & $1.2 \pm 0.3[\underline{\underline{2}}]$ & 1.1 \\
\hline 10 & 0.79 & 150 & 2.5 & 20 & 40 & 0.81 & $2.0 \pm 0.3[2]$ & 2.0 \\
\hline 13 & 0.79 & 150 & 2.5 & 30 & 40 & 0.36 & $1.5 \pm 0.3[\underline{2}]$ & 1.4 \\
\hline 15 & 0.79 & 150 & 2.5 & 30 & 40 & 0.37 & $1.7 \pm 0.3[\underline{\underline{2}}]$ & 1.6 \\
\hline 100 & 0.8 & 100 & 2.5 & 3 & 40 & 14.2 & $22-24[\underline{\underline{9}}]$ & 22.6 \\
\hline 100 & 0.8 & 100 & 2.5 & 6 & 40 & 8.6 & $17-19[9]$ & 17.3 \\
\hline 100 & 0.8 & 100 & 2.5 & 10 & 40 & 5.13 & $11-17[\underline{9}]$ & 13.2 \\
\hline 100 & 0.8 & 100 & 2.5 & 25 & 40 & 0.897 & $6-7[9]$ & 5.0 \\
\hline
\end{tabular}

been compared with experiments for $L \leq 1 \mu \mathrm{m}$. However, the laser absorption efficiency for the target of arbitrary thickness has been calculated by particle-in-cell (PIC) simulations [3] , although there is no analytic result of that.

With the simulation results in ref. [3], the small target thickness will lead to reduced absorption if the target deconfines rapidly and becomes transparent before the end of the laser pulse - but the characteristic velocity for this is the sound speed, not the speed of light. If the critical thickness for recirculation is $L_{c}$, the critical thickness for modified absorption should be much smaller than $L_{c}$. Therefore, for thin foils of the thickness, $L \geq 1 \mu \mathrm{m}$, the laser absorption efficiency keeps a constant about $35-50 \%$ approximately with the thickness, $L$. For the target of $3 \mu \mathrm{m}, \eta \approx 40 \%$ and $\theta_{\text {in }}=17^{\circ}$, the maximum proton energy is $22.6 \mathrm{MeV}$ estimated by our model which is consistent with the experimental data, $22-24 \mathrm{MeV}$. The time-dependent electron density, the acceleration field and the ion speed are shown by Figure 1(b). The hot-electron density increases from 0 and reaches the maximum value 1.5 at the time $t_{l}$. Therefore, for $L=3 \mu \mathrm{m}$ and $L_{c}=15 \mu \mathrm{m}$, the hotelectron recirculation does exist and $N(\tau)$ is up to about 1.5 but not 5 as shown by Sentoku et al. [10]. After $t_{l}$, the electron density decreases quickly to half at about $2.2 t_{l}$. The duration of the hot-electron density and field are $2-5 t_{l}$.

The maximum value of $N(\tau)$ is smaller than or equal to 1 as shown by Figure 1 . Figure 1 shows that: the maximum $N(\tau)$ is about 0.79 which is smaller than 1 , therefore there is no hot-electron 
recirculation phenomena for $L \geq L_{c}$; as the time goes to infinite, the velocity of protons is finite and the maximum energy is about $1.1 \mathrm{MeV}$ while the experimental data is $1.2 \pm 0.3 \mathrm{MeV}$; the duration of the hot-electron density is about $3.6 t_{l}$. Therefore, the duration of the field at the ion front is about $2 t_{l}$, which is consistent with the simulation result[3].

\section{SCALING LAW AND DISCUSSION}

The laser intensity in our model is assumed to be a constant value in the pulse duration. Under this assumption and for a fixed laser energy, the dependence of maximum ion energy on the laser pulse duration is easy to be obtained. There is an optimum pulse duration for the target normal sheath acceleration of ions if the laser energy, focus radius and absorption efficiency sustain constants. It is a conflict of large acceleration gradient and long efficient acceleration time. For long pulse duration, the intensity will be low and the acceleration field will be low. For a high intensity, the efficient acceleration time will be short. Therefore, there is an optimum pulse duration in the ion acceleration.

For different focus radius and target thickness, the dependence of maximum ion energy, $E_{\max , i}$, on pulse duration can be also obtained easily with our model. The results may be different since the plasma density changes with $r_{L}$ and $L$ as shown by Eq. (2) and Eq. (3). The wave length will not influence the dependence of $E_{\max , i}$ on pulse duration.

For a series of given pulse duration, the dependence of $E_{\text {max }, i}$ on laser intensity has also been obtained and the scaling law is given by:

$$
E_{\text {max }, i}=\left\{\begin{array}{l}
\exp \left(a_{1}\right)\left(I_{10^{18} \mathrm{~W} / \mathrm{cm}^{2}} \lambda_{\mu \mathrm{m}}^{2}\right)^{b_{1}}, I_{10^{18} \mathrm{~W} / \mathrm{cm}^{2}} \lambda_{\mu \mathrm{m}}^{2} \geqq 6.4, \\
\exp \left(a_{2}\right)\left(I_{10^{18} \mathrm{~W} / \mathrm{cm}^{2}} \lambda_{\mu \mathrm{m}}^{2}\right)^{b_{2}}, I_{10^{18} \mathrm{~W} / \mathrm{cm}^{2}} \lambda_{\mu \mathrm{m}}^{2} \geqq 6.4,
\end{array}\right.
$$

where $a_{1}, a_{2}, b_{1}, b_{2}$ are all coefficients and shown by Fig. 2 ,

With Eq. (8) and Fig. 2, two important results can be obtained. First, the scaling law is different from the previous results $I^{1 / 2}$. The index $b_{1}$ and $b_{2}$ depend on the laser pulse duration and decrease with pulse duration. It shows the adiabatic expansion of plasmas is also a very important acceleration process and should not be neglected, although they [2, 5] can consist with experiments considering the isothermal expansion only through adjusting of the parameters: $\eta$, the acceleration time $t_{a c c}$, the plasma density $n_{e}$, the opening angle of electrons and so on. Second, the influence of hot-electron recirculation on the ion acceleration can be shown by Fig. 2 approximately. 


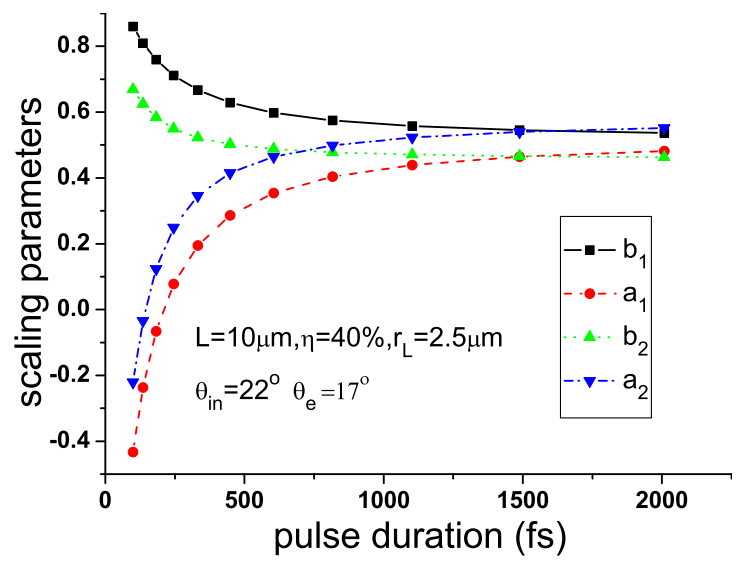

FIG. 2: (Color online) The coefficients in the scaling law given by Eq. 8 versus laser intensity, I. Laser absorption efficiency is assumed $40 \%$, since the target thickness is large enough. Here, focus radius is $2.5 \mu \mathrm{m}$.

With our model, the dependence of maximum ion energy on target thickness is given by:

$$
E_{\text {max }, i}=y_{0}+A \exp \left(-\frac{L-L_{0}}{L_{s}}\right)
$$

for $\eta=40 \%, r_{L}=2.5 \mu \mathrm{m}, \theta_{i n}=22^{\circ}, t_{l}=100 \mathrm{fs}$ and $\theta_{e}=17^{\circ}$, where $y_{0}, A, L_{0}, L_{s}$ are all coefficients. The dependence of them on laser intensity have been shown in Fig. 3, Eq. (9) shows the maximum ion energy decreases with the target thickness in the negative exponential form for a fixed laser absorption and a given laser intensity. However, the laser absorption tends to zero as $L \rightarrow 0$, therefore, the maximum ion energy tends to zero in fact. From Fig. 3, maximum ion energy increases with the laser intensity since the coefficients $y_{0}, A$ all increase with the laser intensity.

In fact, focus radius influences the electron density and then the ion acceleration. For $L=$ $10 \mu \mathrm{m}, \eta=40 \%, \theta_{i n}=22^{\circ}, \theta_{e}=17^{\circ}$, using our model, the effect of focus radius satisfies:

$$
E_{\text {max }, i}=A_{2}+\frac{A_{1}-A_{2}}{1+\left(r_{L} / r_{0}\right)^{p}}
$$

where $A_{1}, A_{2}, r_{0}$ and $p$ are all coefficients and change with laser intensity and shown by Fig. 4 . With Eq. (10), some interesting results can be achieved:

(1) The smaller laser intensity, the larger the critical value $r_{0}$ and the index $p$. Therefore, for $r_{L} \gg r_{0}$ and $\left(r_{L} / r_{0}\right)^{p} \gg 1, E_{\max , i} \approx-\left(r_{L} / r_{0}\right)^{-p}$. The derivation of $E_{\max , i}: d E_{\max , i} / d\left(r L / r_{0}\right)=$ $p\left(r_{L} / r_{0}\right)^{-p-1}$, reflects the rate of change of $E_{\text {max }, i}$ to the focus radius. The rate of change is positive but decreases with focus radius. For larger laser intensity, the larger the rate of change. 


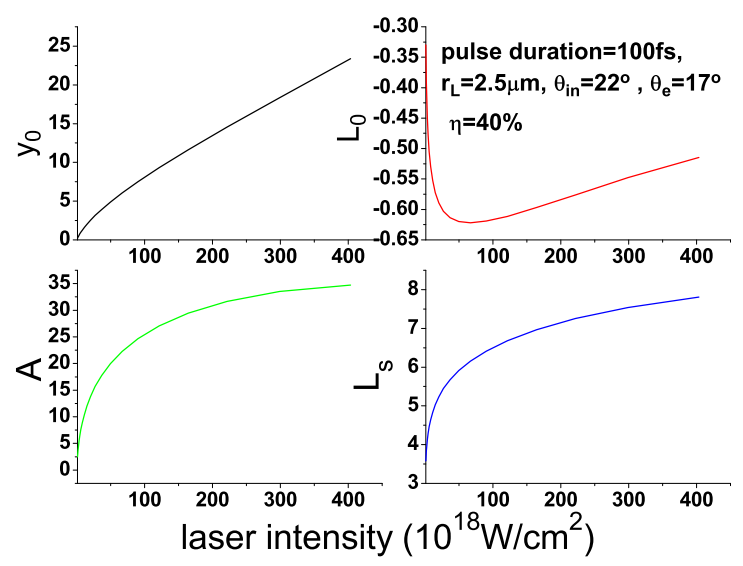

FIG. 3: (Color online) The coefficients of the scaling law of maximum ion energy with respect to target thickness given by Eq. (9) versus laser intensity for $r_{L}=2.5 \mu \mathrm{m}, \eta=40 \%, t_{l}=100 \mathrm{fs}$ and $\lambda=0.8 \mu \mathrm{m}$.

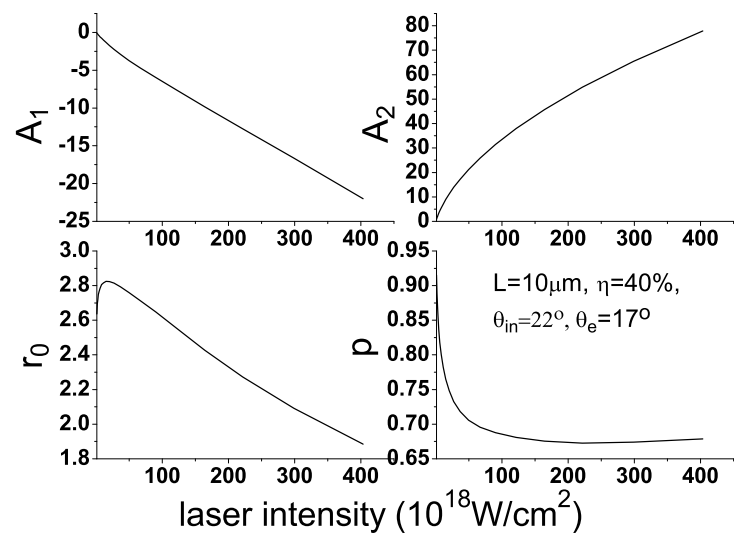

FIG. 4: (Color online) The coefficients of the scaling law of maximum ion energy with respect to focus radius given by Eq. (10) versus laser intensity for $L=10 \mu \mathrm{m}, \eta=40 \%, t_{l}=100 \mathrm{fs}$ and $\lambda=0.8 \mu \mathrm{m}$.

(2) Oppositely, for $r_{L} \ll r_{0}$ and $\left(r_{L} / r_{0}\right)^{p} \ll 1, E_{\max , i} \approx$ constant, which shows the influence of $r_{L}$ on the maximum ion energy can be ignored in this case. For larger laser intensity, the smaller critical focus radius. Therefore, the larger laser intensity, the more sensitive the ion acceleration to the change of focus radius.

The influence of opening angle of hot electrons on maximum ion energy has been discussed in detail by Huang and co-workers in ref. [11]. However, the dilution of the electron density as electrons circulate and spread laterally was not contained there. This effect is considered here with the factor, $F_{\theta, 3-D}$ in Equations (4)-(7). Therefore, the maximum value of electron density here is 1.5 (in Fig. [1(b)) while it is about 5 in ref. [11] and ref. [10]. For the target of arbitrary thickness, the maximum energy of ions heated by target normal sheath acceleration (TNSA) can be obtained 
by this model if the absorption efficiency of laser pulse is given.

Here we will discuss the limits of our model. The prepulse is not considered in our model and the contrast is assumed large about $10^{8}$ which can be achieved in lots of experiments. However, the exist of a prepulse would generate a preplasma and the preplasma size, the scaling length of the preplasma, will most influence the mechanisms of laser absorption and then the temperature of hot electrons. Different laser absorption mechanism results in different generation rate of hot electrons, $f$, and different hot-electron temperature, $T_{e}$. No matter what the mechanisms are, the generation of hot electrons is cumulative and the assumption: $f=\bar{f}$ causes little error relative to that caused by the measurement in experiments. Whatever the temperature of hot electrons is, our model is still in use with the actual temperature instead of the value, $m c^{2}(\gamma-1)$. The laser intensity in our model is assumed to be a constant value in the pulse duration. In fact, the intensity is changing with time and the distribution is about Gaussian distribution. However, the actual intensity distribution with respect to time and position when laser pulse is acting on a target is quite difficult to be measured in real time. Since we do not consider the time distribution of laser intensity, we can not give an estimation of the error. In the next paper, we will consider that case.

\section{CONCLUSION}

In conclusion, the time-dependent isothermal expansion and adiabatic expansion for the target normal sheath proton acceleration is discussed. A two-phase model and a new scaling law of the maximum energy of proton beams have been proposed. The influence of the hot-electron recirculation on the ion acceleration has been accounted in. For $L \geq L_{c}$, the hot-electron recirculation can be ignored. But for $L \leq L_{c}$, the hot-electron recirculation exists and enhances as the target thickness decreases. The results given by our model have been compared with experiments and shown in Table I. The dependence of maximum ion energy on target thickness, focus radius, laser pulse duration have been obtained and shown by equations (8), (9), (10), and Figures 2, 3, 4) and so on. At last, the application and limits of our model has been discussed.

However, for thin foils, the laser absorption efficiency is an important parameter for our model and is still a challenge for this problem. The generation rate of hot electrons in the interaction of laser pulses with the plasma at the front of the target, $f$, is also a challenge. An interesting work that may be considered nest is the time-dependent laser pulse intensity in order to optimize our model further more. 
This work was supported by the Key Project of Chinese National Programs for Fundamental Research (973 Program) under contract No. $2006 C B 806004$ and the Chinese National Natural Science Foundation under contract No. 10334110.

[1] S. C. Wilks, A. B. Langdon, T. E. Cowan, M. Roth, M. Singh, S. Hatchett, M. H. Key, D. Pennington, A. MacKinnon, and R. A. Snavely, Phys. Plasma 8, 542 (2001); L. O. Silva, M. Marti, J. R. Davies, R. A. Fonseca, C. Ren, F. S. Tsung, and W. B. Mori, Physical Review Letters 92, 015002 (2004); Y. Oishi, T. Nayuki, T. Fujii, Y. Takizawa, X. Wang, T. Yamazaki, K. Nemoto, T. Kayoiji, T. Sekiya, K. Horioka, Y. Okano, Y. Hironaka, K. G. Nakamura, K. Kondo and A. A. Andreev, Phys. Plasma 12, 073102 (2005); H. Schwoerer, S. Pfotenhauer, O. Jackel, K. U. Amthor, B. Liesfeld, W. Ziegler, R. Sauerbrey, K. W. D. Ledingham, and T. Esirkepov, Nature 439, 445 (2006); M. Murakami, and M. M. Basko, Phys. Plasma 13, 012105 (2006).

[2] M. Kaluza, J. Schreiber, M. I. K. Santala, G. D. Tsakiris, K. Eidmann, J. Meyer-ter-Vehn, and K. J. Witte, Phys. Rev. Lett 93, 045003 (2004).

[3] E. d'Humieres, E. Lefebvre, L. Gremillet, and V. Malka, Phys. Plasma 12, 062704 (2005).

[4] J. Schreiber, F. Bell, F. Gruner, U. Schramm, M. Geissler, M. Schnurer, S. Ter-Avetisyan, B. M. Hegelich, J. Cobble, E. Brambrink, J. Fuchs, P. Audebert, and D. Habs, Phys. Rev. Lett. 97, 045005 (2006).

[5] J. Fuchs, P. Antici, E. d'Humieres, E. Lefebvre, M. Borghesi, E. Brambrink, C. A. Cecchetti, M. Kaluza, V. Malka, M. Manclossi, S. Meyroneinc, P. Mora1, J. Schreiber, T. Toncian, H. Ppin and P. Audebert, Nature Physics 2, 48 (2006).

[6] L. Robson, P. T. Simpson, R. J. Clarkei, K. W. D. Ledingham, F. Lindau, O. Lundh, T. McCanny, P. Mora, D. Neely, C.-G. Wahlstrom, M. Zepf, and P. McKenna, Nature Physics 3, 58 (2007).

[7] P. Mora, Phys. Rev. Lett. 90, 185002 (2003).

[8] P. Mora, Phys. Rev. E 72, 056401 (2005).

[9] A. J. Mackinnon, Y. Sentoku, P. K. Patel, D. W. Price, S. Hatchett, M. H. Key, C. Andersen, R. Snavely, and R. R. Freeman, Phys. Rev. Lett 88, 215006 (2002).

[10] Y. Sentoku, T. E. Cowan, A. Kemp, and H. Ruhl, Phys. Plasma 10, 2009 (2003).

[11] Y. S. Huang, X. F. Lan, X. J. Duan, Z. X. Tan, N. Y. Wang, Y. J. Shi, X. Z. Tang, and Y. X. He, Phys. Plasmas, 14, 103106 (2007). 
[12] J. J. Santos, F. Amiranoff, S. D. Baton, L. Gremillet, M. Koenig, E. Martinolli, M. Rabec Le Gloahec, C. Rousseaux, D. Batani, A. Bernardinello, G. Greison, and T. Hall, Phys. Rev. Lett 89, 025001 (2002).

[13] R. A. Snavely, M. H. Key, S. P. Hatchett, T. E. Cowan, M. Roth, T. W. Phillips, M. A. Stoyer, E. A. Henry, T. C. Sangster, M. S. Singh, S. C. Wilks, A. MacKinnon, A. Offenberger, D. M. Pennington, K. Yasuike, A. B. Langdon, B. F. Lasinski, J. Johnson, M. D. Perry, and E. M. Campbell, Phys. Rev. Lett 85, 2945 (2000).

[14] D. S. Dorozhkina and V. E. Semenov, Phys. Rev. Lett. 81, 2691 (1998); A. V. Baitin and K. M. Kuzanyan, J. Plasma Phys. 59, 83 (1998); V. F. Kovalev, V. Yu. Bychenkov, and V. T. Tikhonchuk, JETP 95, 226 (2002); V. F. Kovalev and V. Yu. Bychenkov, Phys. Rev. Lett. 90, 185004 (2003).

[15] M. A. True, J. R. Albritton, and E. A. Williams, Phys. Fluids 24, 1885 (1981).

[16] G. Manfredi, S. Mola, and M. R. Feix, Phys. Fluids B 5, 288 (1993). 